\title{
JOINT RECONSTRUCTION OF COMPRESSIVELY SENSED ULTRASOUND RF ECHOES BY EXPLOITING TEMPORAL CORRELATIONS
}

\author{
George Tzagkarakis ${ }^{1}$, Alin Achim ${ }^{2}$, Panagiotis Tsakalides ${ }^{3}$ and Jean-Luc Starck ${ }^{1}$ \\ ${ }^{1}$ CEA, DSM/SEDI/LCS, Centre de Saclay, F-91191 Gif-Sur-Yvette cedex, France \\ ${ }^{2}$ Visual Information Lab, University of Bristol, UK \\ ${ }^{3}$ Dept. of Computer Science, University of Crete \& Institute of Computer Science - FORTH, Greece \\ e-mail: \{georgios.tzagkarakis, jstarck\}@cea.fr, Alin.Achim@bristol.ac.uk,tsakalid@ics.forth.gr
}

\begin{abstract}
In this paper, the principles of compressive sensing are exploited for the joint reconstruction of an ensemble of biomedical ultrasound RF echoes, using a highly reduced set of random measurements. Temporal correlations between the distinct RF echoes are taken into account during the reconstruction, which results in a reduction of the required number of measurements, while also increasing the reconstruction quality. The efficiency of recent state-of-the-art methods is evaluated on a set of real ultrasound data, to highlight the importance of accounting for temporal correlations during reconstruction. Our experimental evaluation reveals an improved performance, both visually and in terms of quality metrics, such as the SSIM and PSNR, when such correlations are extracted during the joint reconstruction of RF echoes, compared with previous methods based on the separate recovery of each $\mathrm{RF}$ echo.
\end{abstract}

Index Terms - Compressive sensing, joint signal reconstruction, structured sparsity, ultrasound RF echoes.

\section{INTRODUCTION}

Ultrasound imaging is among the most widely used cross-sectional medical imaging modalities, mainly because ultrasound has a number of potential advantages, which foster its popularity over other medical imaging techniques. Specifically, ultrasound imaging systems are non-invasive, versatile, they do not use ionizing radiation, and they can be embedded in relatively low-cost portable devices, which operate in real time. However, this real-time nature may be limited by the available acquisition time, or the high volume of the acquired data. Moreover, introduction of new technologies, such as 4D ultrasound, entailing orders of magnitude greater requirements for data transfer, processing, and storage, imposes even greater constraints necessitating the design of efficient data reduction methods.

The general principle of ultrasound image formation involves the transmission of a train of pulses from an array of transducers towards the plane being scanned. The returning echoes are then processed in order to generate an image that displays their location and amplitude. Image compression is necessary in order to reduce the data volume, especially in case of portable ultrasound systems with limited processing, memory, and bandwidth resources, and to achieve a low bit rate, ideally without any perceived loss of image quality. The need for transmission bandwidth and storage capacity in the digital radiology environment, especially in telemedicine appli-

This work was partly supported by CS-ORION Marie Curie IndustryAcademia Partnerships and Pathways (IAPP) project funded by the European Commission in FP7 (PIAP-GA-2009-251605). cations, and the continuous diversification of ultrasound applications keep placing new limitations on the capabilities of existing systems.

In this work, the principles of compressive sensing (CS) $[1,2]$ are exploited to overcome the limitations of traditional ultrasound imaging systems, while providing a more general framework for ultrasound image analysis and extraction of meaningful information. In particular, the original full-resolution ultrasound data are mapped in a much lower-dimensional space, namely, the space of random incoherent measurements. Our goal then is to recover the original ultrasound image given this highly reduced set of measurements.

In a recent work [3], we introduced a novel framework for CS of biomedical ultrasonic signals based on modeling data with symmetric alpha-stable distributions. Then, we proposed an $\ell_{p}$ norm-based minimization approach that employed the iteratively reweighted least squares (IRLS) algorithm, but in which the parameter $p$ was judiciously chosen by relating it to the characteristic exponent of the underlying alpha-stable distributed data. The results showed a significant increase of the reconstruction quality when compared with previous $\ell_{1}$ minimization algorithms. The effect of the random sampling pattern on the reconstruction quality, when working in the frequency domain (k-space) was studied in [4], which was then exploited in [5] for the design of a Bayesian CS algorithm, whose main benefit is that it does not require any hyperparameter adjustment and allows the sparsity level in the Fourier domain to be estimated. However, none of these studies takes into account potential temporal correlations between RF echoes.

Sparse Bayesian learning (SBL) is an important class of Bayesian algorithms. A recent study [6], first introduced SBL for sparse signal recovery using multiple measurement vectors (MSBL), which was then extended in [7] in a block SBL framework accounting for temporal correlations between the individual source signals (T-MSBL).

In this work, we exploit the capability of T-MSBL to extract such correlations on real ultrasound data, resulting in a reduction of the required number of measurements, while attaining an increased reconstruction quality. This method is applied in the time-domain directly, based on the observation that the recorded RF echoes are sparse by themselves, meaning that the effective support consists of only a few high-amplitude values corresponding to pulses reflected from "objects" placed at specific distances from the transducer.

The rest of the paper is organized as follows: Section 2 introduces the sampling process for the simultaneous sensing and compression of the time-domain RF echoes. Section 3 describes the joint reconstruction of the original ultrasound signals by considering temporal correlations, while Section 4 presents an experimental evaluation and comparison with previous methods using real data. Finally, Section 5 concludes, and gives directions for further improvements. 


\section{SAMPLING AND COMPRESSION OF RF ECHOES}

CS is concerned with simultaneously sampling and compressing signals in a more parsimonious fashion, acquiring only the relevant information, rather than first sampling and then compressing separately. The core concept of this theory is that, given an exactly sparse or compressible signal, a highly reduced set of linear random projections contains all the sufficient information to perform the processing of interest, such as, reconstruction, detection, or classification.

In terms of signal approximation, it has been demonstrated [1,2] that if a signal of length $N$ is $K$-sparse in an appropriate dictionary or transform basis, then it can be recovered with high probability from $M=\mathcal{O}\left(K \log \frac{N}{K}\right)$ non-adaptive linear projections onto a measurement basis, which is sufficiently incoherent with the sparsifying dictionary or transform basis. Common choices of measurement bases satisfying this condition, are random matrices with independent, identically distributed (i.i.d.) Gaussian or Bernoulli entries.

In the following, let $\mathbf{X} \in \mathbb{R}^{N \times S}$ denote an ultrasound image, which can be considered as an ensemble of $S$ distinct RF echoes of length $N, \mathbf{X}_{i} \in \mathbb{R}^{N}, i=1, \ldots, S$. Then, a vector of $M$ compressed measurements is generated for the $i$-th RF echo as follows:

$$
\mathbf{y}_{i}=\boldsymbol{\Phi}_{i} \mathbf{X}_{i},
$$

where $\boldsymbol{\Phi}_{i} \in \mathbb{R}^{M \times N}$, with $M<N$, is the measurement matrix. Although, in general, a different measurement matrix can be used for each RF echo, for simplicity we consider that the same matrix is used to acquire all the compressed RF echoes, that is, $\boldsymbol{\Phi}_{i} \equiv \boldsymbol{\Phi}$, $i=1, \ldots, S$. By doing this, a set of compressed measurements is generated directly for the whole ensemble of the one-dimensional (1D) RF echoes as follows:

$$
\mathbf{Y}=\boldsymbol{\Phi} \mathbf{X}
$$

where $\mathbf{Y} \in \mathbb{R}^{M \times S}$ is the matrix of random measurements.

Then, the problem to be solved is stated as follows: Given the low-dimensional matrix of random measurements $\mathbf{Y}$ for the ensemble of $1 D$ RF echoes, along with the measurement matrix $\mathbf{\Phi}$, perform a joint reconstruction of the original high-resolution ultrasound data $\mathbf{X}$ by exploiting potential temporal correlations between the individual RF echoes (columns of $\mathbf{X}$ ).

\section{JOINT RECONSTRUCTION OF RF ECHOES EXPLOITING TEMPORAL CORRELATIONS}

The formulation given by (2) is equivalent to a multiple measurement vectors (MMV) problem, with temporal correlations between the elements in each non-zero, or "dominant", row of $\mathbf{X}$. By "dominant" we refer to those rows of $\mathbf{X}$ consisting of the high-amplitude elements, similarly to the notion of compressibility. Previous algorithms did not consider such temporal correlations, which is especially the case with norm-based optimization methods, where the incorporation of temporal correlations is not straightforward.

In [7], the proposed algorithm models and exploits temporal correlations between sources, under a Gaussian assumption for the signal and noise terms. In the following, we summarize the main components of the temporally correlated, MMV-based SBL (T-MSBL) algorithm introduced in [7], and how these are related to the ultrasound RF data of interest.

The algorithm considers the general noisy measurement model,

$$
\mathbf{Y}=\mathbf{\Phi} \mathbf{X}+\mathbf{V},
$$

where $\mathbf{V} \in \mathbb{R}^{M \times S}$ is the noise term consisting of i.i.d. elements. T-MSBL is based on a Gaussian assumption for both the signal (X) and noise (V) components. More specifically, each source, that is, 1D RF echo, is modeled by a Gaussian density,

$$
f_{X}\left(\mathbf{X}_{i}\right) \sim \mathcal{N}\left(\mathbf{0}, \gamma_{i} \boldsymbol{\Sigma}_{i}\right), i=1, \ldots, S
$$

where $\gamma_{i} \geq 0$ is a hyperparameter controlling the degree of sparsity of the $i$-th RF echo. More specifically, when $\gamma_{i}=0$, the corresponding $\mathbf{X}_{i}$ is an all-zeros vector. Moreover, $\boldsymbol{\Sigma}_{i}$ is a positive definite matrix, which captures the inherent correlation structure of $\mathbf{X}_{i}$. Similarly, the elements of the noise matrix are realizations of a Gaussian density, $f_{V}(v) \sim \mathcal{N}(0, \sigma)$, where $\sigma$ is the noise variance. The use of distinct matrices $\boldsymbol{\Sigma}_{i} \neq \boldsymbol{\Sigma}_{j}, i \neq j$, for different RF echoes entails the drawback of overfitting due to potentially limited data and many parameters to be estimated. A way to overcome this problem is to use a unique matrix $\boldsymbol{\Sigma}_{i} \equiv \boldsymbol{\Sigma}, \forall i=1, \ldots, S$, to model the correlations of all the RF echoes up to a non-negative scalar $\gamma_{i}$.

Following a maximum a posteriori (MAP) estimation methodology, the jointly reconstructed ensemble of RF echoes is simply the mean of the posterior distribution of $\mathbf{X}$, which is also a Gaussian. In each step of T-MSBL, the parameters to be updated are the hyperparameters $\left\{\gamma_{i}\right\}_{i=1}^{S}$, the matrix $\boldsymbol{\Sigma}$, and the noise variance $\sigma$. The model parameters of the posterior Gaussian distribution, along with the updating rules for the above parameters are as follows (where the superscript $t$ is used to denote the current estimate of a given variable, and $t-1$ its estimate in the previous iteration):

$$
\begin{aligned}
\boldsymbol{\Sigma}_{x}^{t} & =\left(\left(\boldsymbol{\Gamma}^{t-1}\right)^{-1}+\frac{1}{\sigma^{t-1}} \boldsymbol{\Phi}^{T} \boldsymbol{\Phi}\right)^{-1} \\
\mathbf{X}^{t} & =\boldsymbol{\Gamma}^{t-1} \boldsymbol{\Phi}^{T}\left(\sigma^{t-1} \mathbf{I}+\boldsymbol{\Phi} \boldsymbol{\Gamma}^{t-1} \boldsymbol{\Phi}^{T}\right)^{-1} \mathbf{Y} \\
\gamma_{i}^{t} & =\frac{1}{N} \mathbf{X}_{i}^{t}\left(\boldsymbol{\Sigma}^{t-1}\right)^{-1} \mathbf{X}_{i}^{t T}+\left(\boldsymbol{\Sigma}_{x}^{t}\right)_{i i}, \forall i=1, \ldots, S \\
\hat{\boldsymbol{\Sigma}}^{t} & =\sum_{i=1}^{S} \frac{\mathbf{X}_{i}^{t T} \mathbf{X}_{i}^{t}}{\gamma_{i}^{t}}+\eta \mathbf{I} \\
\boldsymbol{\Sigma}^{t}= & \frac{\hat{\boldsymbol{\Sigma}}^{t}}{\left\|\hat{\boldsymbol{\Sigma}}^{t}\right\|_{F}} \\
\sigma^{t}= & \frac{1}{M N}\left\|\mathbf{Y}-\mathbf{\Phi} \mathbf{X}^{t}\right\|_{F}^{2}+ \\
& \frac{\sigma^{t}}{M} \operatorname{Tr}\left[\mathbf{\Phi} \boldsymbol{\Gamma}^{t} \boldsymbol{\Phi}^{T}\left(\sigma^{t} \mathbf{I}+\mathbf{\Phi} \boldsymbol{\Gamma}^{t} \boldsymbol{\Phi}^{T}\right)^{-1}\right]
\end{aligned}
$$

where $\boldsymbol{\Gamma}=\operatorname{diag}\left(\gamma_{1}, \ldots, \gamma_{S}\right), \eta>0$ is a regularization parameter, which is used especially in low and medium SNR cases to increase the robustness due to estimation errors of $\gamma_{i}$ and $\mathbf{X}_{i}$, and $\|\cdot\|_{F}$ denotes the Frobenius norm of a matrix. The algorithm terminates when a predefined maximum number of iterations, $T_{\max }$ is reached, or the reconstruction error between two consecutive iterations falls below a threshold, $\max _{m, n}\left(\mathbf{X}_{m, n}^{t}-\mathbf{X}_{m, n}^{t-1}\right)<\epsilon\left(\right.$ where $\mathbf{X}_{m, n}$ denotes the element of $\mathbf{X}$ in row $m$ and column $n$ ).

\section{EXPERIMENTAL EVALUATION}

In this section, the performance of reconstructing jointly an RF echoes ensemble by accounting for temporal correlations is evaluated for T-MSBL, and compared with recent CS methods which do not exploit explicitly temporal correlations. More specifically, all the methods are tested on a set of three real ultrasound images of phantoms, shown in Fig. 1, with each image being sampled and compressed simultaneously using a measurement matrix $\boldsymbol{\Phi}$ generated as the orthonormal basis of a Gaussian matrix G. The orthonormalization simply ensures that $\boldsymbol{\Phi}^{T} \boldsymbol{\Phi}=\mathbf{I}$, which makes the numerical implementation more robust by avoiding the lack of an exact inverse 

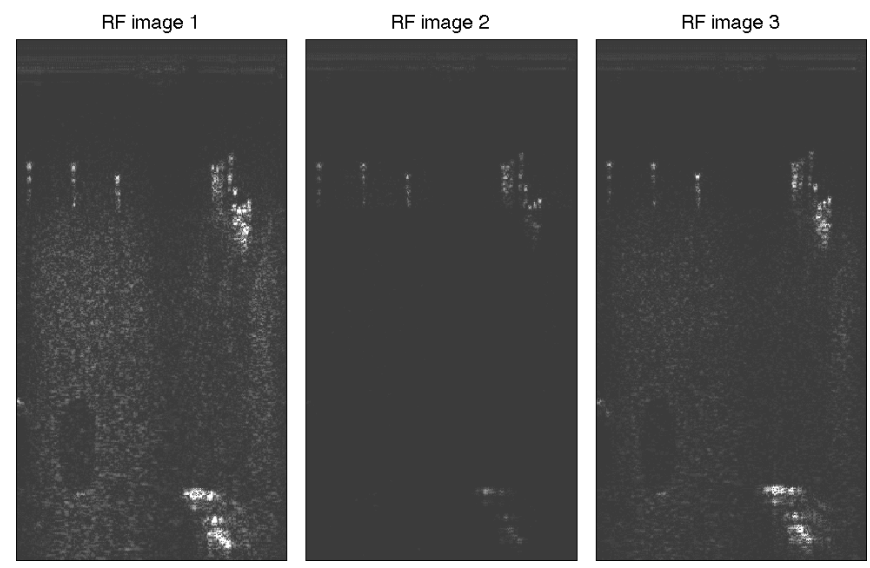

Fig. 1. Real ultrasound images used in the experimental evaluation.

matrix, while maintaining the same column space with the original random matrix $\mathbf{G}$.

The ultrasound images used consist of 256 1D RF echoes, each one of size $N=1032$. The number of compressed measurements, $M$, varies as a percentage of the full dimension, $M=\rho \cdot N$, with $\rho \in\{0.10,0.20,0.30,0.40\}$. T-MSBL initializes with $\Gamma^{0}=\mathbf{I}$, $\sigma^{0}=10^{-3}$, and terminates for $T_{\max }=2000$. The threshold $\epsilon=$ $10^{-6}$, and the regularization parameter $\eta=2$. The performance achieved by T-MSBL is compared against the following algorithms:

i) IRLS-prior: the 1D RF echoes are reconstructed separately using an iterative re-weighted least squares (IRLS) algorithm with prior information [8]. An initial "guess" of the effective sparse support is used as prior information, which corresponds to the indices of the $K$ highest amplitude elements of each RF echo. Although this choice corresponds to an idealcase scenario, where the full-resolution RF echoes have been recorded and the true support is known, it will be used as a benchmark to compare with. In the subsequent evaluation we set $K=\operatorname{round}(c \cdot N)$ with $c=10 \%$.

ii) OMP: each $1 \mathrm{D} \mathrm{RF}$ echo is reconstructed using the orthogonal matching pursuit algorithm [9].

iii) S $\alpha$ S-IRLS: each RF echo is recovered using our probabilistic algorithm introduced in [3], an $\ell_{2} p$ re-weighted method which is based on modeling the RF echoes using symmetric alpha-stable distributions.

iv) ISAL1: each RF echo is reconstructed separately using a recent infeasible-point subgradient algorithm for $\ell_{1}$ - minimization [10], which is faster and more accurate when compared with several of the previous $\ell_{1}$-based methods.

An analysis of the results is undertaken in terms of reconstruction quality, which is measured by means of the structural similarity index (SSIM) [11] and peak signal-to-noise ratio (PSNR) of the reconstructed echoes ensemble compared with the original ensemble. SSIM resembles more closely the human visual perception, and as such, it is often preferred than the commonly used PSNR. For a given image I and its reconstruction I the SSIM is defined by:

$$
\operatorname{SSIM}=\frac{\left(2 \mu_{\mathrm{I}} \mu_{\hat{\mathrm{I}}}+c_{1}\right)\left(2 \sigma_{\hat{\mathrm{I}}}+c_{2}\right)}{\left(\mu_{\mathrm{I}}^{2}+\mu_{\hat{\mathrm{I}}}^{2}+c_{1}\right)\left(\sigma_{\mathrm{I}}^{2}+\sigma_{\hat{\mathrm{I}}}^{2}+c_{2}\right)},
$$

where $\mu_{\mathrm{I}}, \sigma_{\mathrm{I}}$ are the mean and standard deviation of I (similarly for $\hat{\mathrm{I}}), \sigma_{\text {II }}$ denotes the correlation coefficient of the two images, and

$c_{1}, c_{2}$ stabilize the division with a weak denominator. In particular, when SSIM equals 0 the two images are completely distinct, while when the two images are matched perfectly SSIM is equal to 1 .

Table 1 shows both the SSIM and the PSNR (in parentheses) between the original RF images and their reconstructions using IRLSprior, OMP, S $\alpha$ S-IRLS, ISAL1, and T-MSBL, for a varying sampling rate. As it can be seen, for all the ultrasound images the best performance, excluding the ideal scenario of IRLS-prior, is observed for T-MSBL, verifying the importance of taking into account the presence of temporal correlations. As we mentioned before, the results for IRLS-prior are given as a benchmark to compare with, since in practice the partial or the exact prior knowledge of the true sparse support will be unavailable, especially if the RF echoes are recorded directly in the compressed measurements domain. Despite this fact, we observe that there exist cases (e.g., reconstruction results for RF image 2), where T-MSBL outperforms IRLS-prior even without any prior knowledge of the original sparse support.

Moreover, as expected, the reconstruction quality of all methods increases by increasing the sampling rate. Focusing on T-MSBL, there are cases, such as for RF images 1 and 3, where T-MSBL is outperformed slightly by other methods when the sampling rate is fixed at $10 \%$. This is simply because the accuracy in estimating the covariance matrices, and subsequently, the hyperparameters $\left\{\gamma_{i}\right\}_{i=1}^{S}$ (cf. (5)-(11)) affecting the performance of T-MSBL, decreases as the sampling rate is reduced. As an overall conclusion, we observe that by exploiting temporal correlations we are able to lower the amount of information needed to achieve the same, or even an improved, reconstruction quality without any prior knowledge of the jointly sparse support.

In addition, a comparison between the SSIM and PSNR values of Table 1, and the ultrasound images shown in Fig. 1, reveals the inefficiency of OMP-based approaches in reconstructing more complex ultrasound images. By "complex", we mean images presenting also noise-like features, apart from the dominant structures, as is the case for RF images 1 and 3, for which OMP achieves inferior performance when compared with the other methods. On the other hand, $\mathrm{S} \alpha \mathrm{S}$-IRLS is faring particularly good by comparison, while the recently introduced ISAL1 algorithm gives similarly faithful results.

For a qualitative analysis, Fig. 2 shows a patch from the original ultrasound RF image 1 , along with its reconstructions using the above five methods, for a sampling rate of $40 \%$. Visually, we observe that IRLS-prior and T-MSBL appear to be the best. This behavior is not surprising for IRLS-prior, since it employs prior knowledge of the original sparse support. As such, less distortion is introduced to the areas corresponding to the indices which do not belong to this initial support. However, this will not be the case in a practical scenario, where we would be interested in reconstructing RF images from their compressed measurements directly, without having access to the original RF echoes. Moreover, OMP and ISAL1 reconstructions introduce some noise in areas where the original patch is clear (e.g., top part of the patch), while $\mathrm{S} \alpha \mathrm{S}$-IRLS reconstruction introduces some noise in areas of high signal energy. In any case, the visual inspection is in agreement with the SSIM and PSNR values obtained (cf. Table 1), indicating the importance of accounting for temporal correlations between the RF echoes during reconstruction, as it is done by T-MSBL.

\section{CONCLUSIONS AND FURTHER EXTENSIONS}

In this work, we tackled the problem of joint reconstruction of an ensemble of RF echoes, by exploiting the presence of temporal correlations. We showed, through experimentation with real ultrasound data, and comparison with recent state-of-the-art reconstruction 


\begin{tabular}{|c|c|c|c|c|c|c|}
\hline \multirow{2}{*}{ RF image } & \multirow{2}{*}{$\frac{M}{N} \%$} & \multicolumn{5}{|c|}{ Method } \\
\hline & & IRLS-prior & $\overline{\mathrm{OMP}}$ & S $\alpha$ S-IRLS & ISAL1 & T-MSBL \\
\hline \multirow{4}{*}{1} & 10 & $\begin{array}{c}0.884 \\
(37.40)\end{array}$ & \begin{tabular}{|c|}
0.724 \\
$(32.40)$
\end{tabular} & $\begin{array}{c}0.866 \\
(35.08)\end{array}$ & $\begin{array}{c}0.833 \\
(35.21)\end{array}$ & $\begin{array}{c}0.850 \\
(35.37)\end{array}$ \\
\hline & 20 & $\begin{array}{c}0.896 \\
(38.20)\end{array}$ & $\begin{array}{c}0.739 \\
(33.99)\end{array}$ & $\begin{array}{c}0.868 \\
(35.62)\end{array}$ & $\begin{array}{c}0.857 \\
(36.87)\end{array}$ & $\begin{array}{c}0.871 \\
(36.80)\end{array}$ \\
\hline & 30 & $\begin{array}{c}0.906 \\
(38.88)\end{array}$ & $\begin{array}{c}0.764 \\
(35.15) \\
\end{array}$ & $\begin{array}{c}0.875 \\
(36.21)\end{array}$ & \begin{tabular}{|c}
0.880 \\
$(38.18)$
\end{tabular} & $\begin{array}{c}0.893 \\
(38.20)\end{array}$ \\
\hline & 40 & $\begin{array}{c}0.917 \\
(39.59)\end{array}$ & \begin{tabular}{|c|}
0.794 \\
$(36.07)$ \\
\end{tabular} & $\begin{array}{c}0.884 \\
(36.85)\end{array}$ & $\begin{array}{c}0.902 \\
(39.37)\end{array}$ & $\begin{array}{c}0.914 \\
(39.44)\end{array}$ \\
\hline \multirow{4}{*}{2} & 10 & $\begin{array}{c}0.993 \\
(53.01)\end{array}$ & $\begin{array}{c}0.982 \\
(47.65)\end{array}$ & $\begin{array}{c}0.990 \\
(48.83)\end{array}$ & $\begin{array}{c}0.991 \\
(50.33)\end{array}$ & $\begin{array}{c}0.992 \\
(50.60)\end{array}$ \\
\hline & 20 & $\begin{array}{c}0.995 \\
(53.83)\end{array}$ & \begin{tabular}{|c|}
0.995 \\
$(50.43)$ \\
\end{tabular} & $\begin{array}{c}0.990 \\
(49.36)\end{array}$ & $\begin{array}{c}0.994 \\
(53.07)\end{array}$ & $\begin{array}{c}0.996 \\
(53.63)\end{array}$ \\
\hline & 30 & $\begin{array}{c}0.996 \\
(54.52)\end{array}$ & $\begin{array}{c}0.994 \\
(52.81)\end{array}$ & $\begin{array}{c}0.992 \\
(49.92)\end{array}$ & $\begin{array}{c}0.996 \\
(55.31)\end{array}$ & $\begin{array}{c}0.997 \\
(55.98)\end{array}$ \\
\hline & 40 & $\begin{array}{c}0.996 \\
(55.32)\end{array}$ & \begin{tabular}{|c|}
0.995 \\
$(54.34)$ \\
\end{tabular} & $\begin{array}{c}0.992 \\
(50.62)\end{array}$ & $\begin{array}{c}0.995 \\
(57.17) \\
\end{array}$ & $\begin{array}{c}0.998 \\
(56.92) \\
\end{array}$ \\
\hline & 10 & $\begin{array}{c}0.954 \\
(42.26)\end{array}$ & \begin{tabular}{|c|}
0.874 \\
$(37.35)$ \\
\end{tabular} & $\begin{array}{c}0.939 \\
(38.49)\end{array}$ & \begin{tabular}{|c|}
0.931 \\
$(39.75)$
\end{tabular} & $\begin{array}{c}0.933 \\
(39.63)\end{array}$ \\
\hline & 20 & $\begin{array}{c}0.959 \\
(43.05)\end{array}$ & \begin{tabular}{|c|}
0.889 \\
$(39.17)$
\end{tabular} & $\begin{array}{c}0.938 \\
(39.06)\end{array}$ & $\begin{array}{c}0.944 \\
(41.82) \\
\end{array}$ & $\begin{array}{c}0.946 \\
(41.90)\end{array}$ \\
\hline & 30 & $\begin{array}{c}0.963 \\
(43.70)\end{array}$ & $\begin{array}{c}0.907 \\
(40.52) \\
\end{array}$ & $\begin{array}{c}0.939 \\
(39.62)\end{array}$ & $\begin{array}{c}0.956 \\
(43.51) \\
\end{array}$ & $\begin{array}{c}0.957 \\
(43.70)\end{array}$ \\
\hline & 40 & $\begin{array}{c}0.967 \\
(44.47)\end{array}$ & $\begin{array}{c}0.921 \\
(41.53)\end{array}$ & $\begin{array}{c}0.942 \\
(40.26)\end{array}$ & $\begin{array}{c}0.964 \\
(44.69)\end{array}$ & $\begin{array}{c}0.966 \\
(44.79)\end{array}$ \\
\hline
\end{tabular}

Table 1. Comparison of methods for reconstructing RF echoes ensembles with sampling rate $\frac{M}{N} \in\{10 \%, 20 \%, 30 \%, 40 \%\}$. Values are the SSIM between the original and reconstructed ensembles, along with the corresponding PSNR (in $\mathrm{dB}$ ) given in parentheses. The higher SSIM and PSNR values, the better the reconstruction.

methods, that the extraction of such correlations, and subsequently the joint recovery of the $1 \mathrm{D}$ RF echoes, enhanced the reconstruction quality over CS algorithms, which recover each echo separately.

However, the standard T-MSBL algorithm is designed under a Gaussian assumption for the signal and noise components. Our previous work [3] showed that RF echoes can be modeled accurately using symmetric alpha-stable densities. Thus, the extension of TMSBL by incorporating alpha-stable models could improve the reconstruction quality. A further extension, which is also of high interest, concerns the case of 3D ultrasound data, and more specifically, the design of a framework for object detection, by extracting not only temporal correlations within the same image, but also across different images of the same 3D RF volume.

\section{ACKNOWLEDGEMENTS}

The authors would like to thank Dr. Haidong Liang from Bristol General Hospital for providing the ultrasound data used in this paper.

\section{REFERENCES}

[1] E. Candès, J. Romberg and T. Tao, "Robust uncertainty principles: Exact signal reconstruction from highly incomplete frequency information", IEEE Trans. Inform. Th., Vol. 52, No. 2, pp. 489-509, Feb. 2006.

[2] D. Donoho, "Compressed sensing", IEEE Trans. Inform. Th., Vol. 52, No. 4, pp. 1289-1306, Apr. 2006.

[3] A. Achim, B. Buxton, G. Tzagkarakis and P. Tsakalides, "Compressive sensing for ultrasound RF echoes using alpha-

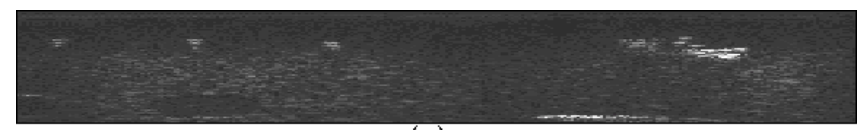

(a)

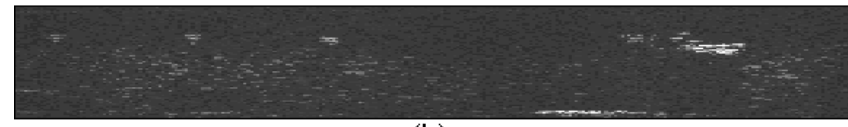

(b)

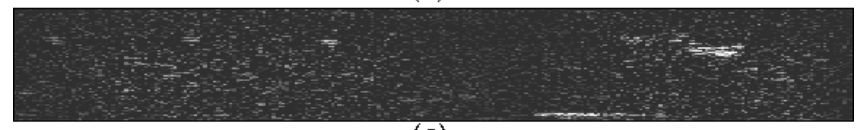

(c)

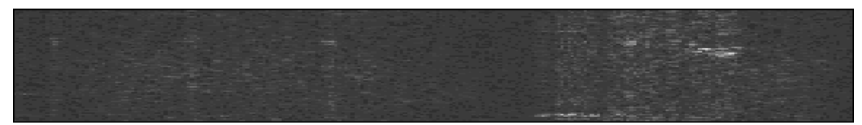

(d)

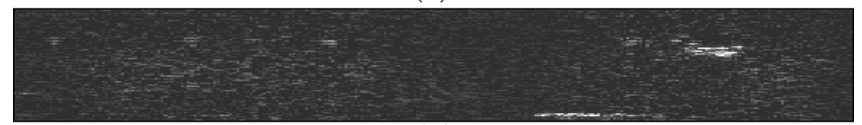

(e)

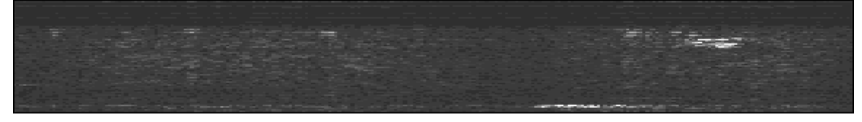

(f)

Fig. 2. Original and reconstructed patches of RF image 1 with sampling rate 40\%: (a) original image, reconstructions with (b) IRLSprior, (c) OMP, (d) S $\alpha$ S-IRLS, (e) ISAL1, and (f) T-MSBL.

stable distributions," in Proc. IEEE Eng. in Med. and Biology Soc. (EMBC '10), Buenos Aires, Argentina, Sep. 1-4, 2010.

[4] C. Quinsac, A. Basarab, J.-M. Girault and D. Kouame, "Compressed sensing of ultrasound images: Sampling of spatial and frequency domains," in Proc. IEEE Work. on Signal Proc. Syst. (SIPS '10), Oct. 2010.

[5] C. Quinsac, N. Dobigeon, A. Basarab, J.-Y. Tourneret and D. Kouame, "Bayesian compressed sensing in ultrasound imaging," in Proc. IEEE Work. Comput. Adv. in Multi-Sensor Adaptive Proc. (CAMSAP '11), Puerto Rico, Dec. 2011.

[6] D. Wipf and B. Rao, "An empirical Bayesian strategy for solving the simultaneous sparse approximation problem, IEEE Trans. Signal Proc., Vol. 55, No. 7, pp. 3704-3716, 2007.

[7] Z. Zhang and B. Rao, "Sparse signal recovery with temporally correlated source vectors using sparse Bayesian learning," IEEE Sel. Topics Sig. Proc., Vol. 5, No. 5, pp. 912-926, 2011.

[8] C. Miosso et al., "Compressive sensing reconstruction with prior information by iteratively reweighted least-squares," IEEE Trans. Signal Proc., Vol. 57, No. 6, pp. 2424-2431, June 2009.

[9] J. Tropp and A. Gilbert, "Signal recovery from random measurements via orthogonal matching pursuit," IEEE Trans. Inform. Th., Vol. 53, No. 12, pp. 4655-4666, Dec. 2007.

[10] D. Lorenz, M. Pfetsch and A. Tillmann, "An infeasible-point subgradient algorithm and a computational solver comparison for $\ell_{1}$-minimization," in Proc. Work. Signal Proc. with Adapt. Sparse Structured Repr., Edinburgh, UK, June 27-30, 2011.

[11] Z. Wang, A. Bovik, H. Sheikh and E. Simoncelli, "Image quality assessment: From error visibility to structural similarity," IEEE Trans. Image Proc., Vol. 13, No. 4, Apr. 2004. 